\title{
Non-unitary Conformal Field Theory and Logarithmic Operators for Disordered Systems
}

\section{Z. Maassarani}

\author{
D. Serban \\ CEA-SACLAY, Service de Physique Théorique \\ F-91191 Gif-sur-Yvette Cedex, FRANCE
}

\begin{abstract}
We consider the supersymmetric approach to gaussian disordered systems like the random bond Ising model and Dirac model with random mass and random potential. These models appeared in particular in the study of the integer quantum Hall transition. The supersymmetric approach reveals an $\operatorname{osp}(2 / 2)_{1}$ affine symmetry at the pure critical point. A similar symmetry should hold at other fixed points. We apply methods of conformal field theory to determine the conformal weights at all levels. These weights can generically be negative because of non-unitarity. Constraints such as locality allow us to quantize the level $k$ and the conformal dimensions. This provides a class of (possibly disordered) critical points in two spatial dimensions. Solving the Knizhnik-Zamolodchikov equations we obtain a set of four-point functions which exhibit a logarithmic dependence. These functions are related to logarithmic operators. We show how all such features have a natural setting in the superalgebra approach as long as gaussian disorder is concerned.
\end{abstract}

April 1996

SPHT-T96/040

hep-th/9605062 


\section{Introduction}

There is growing evidence that some disordered systems at criticality share two unusual features: The existence of logarithmic operators in the spectrum of the theory and the existence of an infinite number of relevant operators with negative conformal dimensions. Logarithmic operators seem to be connected to hidden continuous symmetries. In [1] logarithmic operators were found to generate a change in a coupling constant of the effective WZNW action for $S U(2 r), r \rightarrow 0$, obtained after use of the replica trick. The existence of an infinite number of conformal operators [2] means that there is an infinite number of relevant perturbations which render the critical point unstable. Such a set of conformal dimensions is also related to the phenomenon of multifractality.

Our aim in this paper is to show that both features follow naturally from a supersymmetric treatment of the disorder. We consider the two-dimensional random bond Ising model at criticality and the random Dirac model in $2+1$ dimensions. These gaussian models allow a supersymmetric formulation of averages over disorder [3]. One identifies a global superalgebra symmetry of the effective action and assumes it is enhanced to an affine symmetry at a new critical point [2, 4, as happened at the pure critical point. One then derives the Sugawara stress-energy tensor and obtains the set of conformal dimensions associated with the primary fields. Additional constraints such as locality allow to further restrict the operator content of the theory. This provides a class of, possibly disordered, critical points in two spatial dimensions. The specific structure of the superalgebra implies that such dimensions can generically be negative. The presence of logarithmic operators is also straightforward from the point of view of this algebra. Unlike ordinary Lie algebras, superalgebras have indecomposable (not fully reducible) representations. It is then possible to show on general grounds that such representations imply the existence of logarithmic operators and logarithms in correlation functions.

This paper is organized as follows. In section 2 we briefly recall the supersymmetric approach for the random bond Ising model and the random Dirac model. In section 3 we write down the $\operatorname{sep}(2 / 2)_{k}$ current algebra and find the Sugawara stress-tensor. In section 4 we briefly review the representations of $\operatorname{ssp}(2 / 2)$ and obtain the conformal weights of primary fields. In section 5 we derive the quantization of $k$ from locality constraints. In section 6 we show how indecomposable representations lead to logarithmic operators and consider an atypical representation. In section 7 we obtain some logarithm-dependent four-point functions. We conclude in section 8.

\section{The supersymmetric method for disordered sys- tems}

The supersymmetric method is applicable to models which are gaussian at fixed disorder, but it provides a good starting point for disentangling properties of a large class of disordered conformal field theories. The study of the random bond Ising model reveals that an appropriate algebraic framework for studying gaussian disordered systems at criticality should be based on affine Lie superalgebra with zero superdimension. In the case of the random Ising model this algebra is $\operatorname{osp}(2 N / 2 N)$. The fact that the algebra has an equal number of bosonic and fermionic generators ensures that the Virasoro central charge of 
the Sugawara stress-tensor vanishes. This is needed by construction for a disordered system. The vanishing of the central charge does not imply the triviality of the theory since it is not unitary. The relevance of affine Lie superalgebras was independently realized in refs. [2, 4].

We now briefly recall the supersymmetric method for the study of the random bond Ising model and the random Dirac model. These models have been analyzed using the replica method in refs. [5, 6, 7]. The random bond Ising model has also been studied in the context of massless scattering theories [8].

\section{The random bond Ising model}

In the scaling limit, near criticality, the Ising model is described by a massive real Majorana fermion with mass $\bar{m} \sim \tau=\frac{\left(T_{C}-T\right)}{T_{C}}$ were $T_{C}$ is the critical temperature. In the presence of disorder, i.e. when the coupling constants between sites belong to a random set, the mass becomes a function of space position. The random Ising model is defined by the action $(z=x+i y)$ :

$$
S[m(x)]=\int \frac{d^{2} x}{4 \pi}\left(\psi \partial_{\bar{z}} \psi+\bar{\psi} \partial_{z} \bar{\psi}+i m(x) \bar{\psi} \psi\right)
$$

where $\psi$ and $\bar{\psi}$ are grassmanian fields. The mass $m(x)$ is chosen to be a quenched random variable with a gaussian measure:

$$
P[m]=\exp \left(-\frac{1}{4 g} \int \frac{d^{2} x}{2 \pi}(m(x)-\bar{m})^{2}\right) .
$$

The energy operator $\epsilon(x)=i \bar{\psi}(x) \psi(x)$ has dimension one. The Harris criterion tells us that randomness in the bond interaction is marginal in the $2 \mathrm{~d}$ Ising model. It turns out that it is not exactly marginal but only marginally irrelevant. At criticality the disorder only induces logarithmic corrections to the pure system.

In order to compute averages of products of correlation functions one introduces a number of copies of fermions and of their supersymmetric partners equal to the number of correlation functions in the product [3, 4]. One then rewrites these averages as fermionic and bosonic path integrals. For the Ising model with $\bar{m}=0$ one obtains the following effective action for the disorder average of the product of two correlation functions:

$$
\begin{aligned}
S_{\mathrm{eff}} & =\int \frac{d^{2} x}{2 \pi}\left(\psi_{-} \partial_{\bar{z}} \psi_{+}+\bar{\psi}_{-} \partial_{z} \bar{\psi}_{+}+\eta \partial_{\bar{z}} \gamma+\bar{\eta} \partial_{z} \bar{\gamma}\right)+\frac{g}{8} \int \frac{d^{2} x}{\pi} \Phi_{\mathrm{pert}} \\
& =S_{*}+\frac{g}{8} \int \frac{d^{2} x}{\pi} \Phi_{\mathrm{pert}}
\end{aligned}
$$

with

$$
\Phi_{\text {pert }}=\left(\bar{\psi}_{-} \psi_{+}-\psi_{-} \bar{\psi}_{+}+\bar{\eta} \gamma-\eta \bar{\gamma}\right)^{2}
$$

The $\psi$ are complex fermions and $\eta$ and $\gamma$ are complex bosonic fields. This action can be viewed as a perturbation of the (non-unitary) conformal field theory specified by the action $S_{*}$. This fixes the normalization of the fields to be:

$$
\psi_{-}(z) \psi_{+}(w) \sim \frac{1}{z-w} \quad, \quad \gamma(z) \eta(w) \sim \frac{1}{z-w} .
$$


The central charge of the Virasoro algebra is zero. Note that since the fermions $\psi_{ \pm}$have dimension one half the perturbing field $\Phi_{\text {pert }}$ has dimension two. It is therefore marginal.

The conformal field theory specified by $S_{*}$ is invariant under an affine supersymmetric algebra whose conserved currents are:

$$
\begin{array}{rll}
G_{ \pm}(z)=\eta(z) \psi_{ \pm}(z) & , & \widehat{G}_{ \pm}(z)=\gamma(z) \psi_{ \pm}(z) \\
K(z)=\eta^{2}(z) & , & \widehat{K}(z)=\gamma^{2}(z) \\
J(z)=: \psi_{-}(z) \psi_{+}(z): & , & H(z)=: \gamma(z) \eta(z): .
\end{array}
$$

The dots refer to fermionic and bosonic normal ordering. There are four fermionic currents, $G_{ \pm}$and $\widehat{G}_{ \pm}$, which are generators of supersymmetric transformations, and four bosonic ones. They form a representation of the affine $\operatorname{ssp}(2 / 2)$ [12, 13] current algebra at level one.

The perturbing field can also be written as a bilinear in the currents,

$$
\Phi_{\mathrm{pert}}=2\left[\bar{J} J-\bar{H} H+\frac{1}{2}(\bar{K} \widehat{K}+\overline{\widehat{K}} K)+\bar{G}_{-} \widehat{G}_{+}-\overline{\widehat{G}}_{-} G_{+}+\bar{G}_{+} \widehat{G}_{-}-\overline{\widehat{G}}_{+} G_{-}\right] .
$$

In other words the perturbation (雨) is a current-current perturbation. Therefore the action $S_{\text {eff }}$ preserves a global $\operatorname{osp}(2 / 2)$ symmetry.

The random Dirac model

The random model of Dirac fermions has been introduced in connection with the quantum Hall transition [9]. Its action is:

$$
\begin{aligned}
S= & \int \frac{d^{2} x}{2 \pi}\left(\psi_{-} \partial_{\bar{z}} \psi_{+}+\bar{\psi}_{-} \partial_{z} \bar{\psi}_{+}\right. \\
& \left.+i \frac{m(x)}{2}\left(\bar{\psi}_{-} \psi_{+}-\psi_{-} \bar{\psi}_{+}\right)+i \frac{V(x)}{2}\left(\bar{\psi}_{-} \psi_{+}+\psi_{-} \bar{\psi}_{+}\right)\right) .
\end{aligned}
$$

The random variables $m$ and $V$ have a gaussian distribution with widths $g_{M}$ and $g_{V}$. We denote by $\Phi_{M}$ and $\Phi_{V}$ the perturbing fields coupled to the constants $g_{M}$ and $g_{V}$ after averaging over the disorder. In the two-copy sector we can write them in terms of the $\operatorname{osp}(2 / 2)$ currents:

$$
\begin{aligned}
\Phi_{V} & =2 \bar{H} H-2 \bar{J} J+\bar{K} \widehat{K}+\overline{\widehat{K}} K+2 \bar{G}_{-} \widehat{G}_{+}-2 \widehat{\widehat{G}}_{+} G_{-}+2 \widehat{\widehat{G}}_{-} G_{+}-2 \bar{G}_{+} \widehat{G}_{-}, \\
\Phi_{M} & =2 \bar{J} J-2 \bar{H} H+\bar{K} \widehat{K}+\overline{\widehat{K}} K+2 \bar{G}_{-} \widehat{G}_{+}-2 \widehat{\widehat{G}}_{+} G_{-}-2 \widehat{\widehat{G}}_{-} G_{+}+2 \bar{G}_{+} \widehat{G}_{-} .
\end{aligned}
$$

We see that $\Phi_{M}=\Phi_{\text {pert }}$.

Both $\Phi_{M}$ and $\Phi_{V}$ preserve a global osp $(2 / 2)$ symmetry, whose generators are

$$
\begin{array}{rrrr}
H_{0}+\bar{H}_{0}, & J_{0}+\bar{J}_{0}, & K_{0}+\bar{K}_{0}, & \widehat{K}_{0}+\overline{\widehat{K}}_{0}, \\
G_{+0}+\bar{G}_{+0} & \widehat{G}_{+0}+\widehat{\widehat{G}}_{+0}, & G_{-0}+\bar{G}_{-0}, & \widehat{G}_{-0}+\widehat{\widehat{G}}_{-0} .
\end{array}
$$

for the perturbation $\Phi_{M}$, and

$$
\begin{array}{rrrr}
H_{0}+\bar{H}_{0}, & J_{0}+\bar{J}_{0}, & K_{0}-\bar{K}_{0}, & \widehat{K}_{0}-\overline{\widehat{K}}_{0}, \\
G_{+0}+\bar{G}_{+0}, & \widehat{G}_{+0}-\overline{\widehat{G}}_{+0}, & G_{-0}-\bar{G}_{-0}, & \widehat{G}_{-0}+\widehat{\widehat{G}}_{-0} .
\end{array}
$$


for the perturbation $\Phi_{V}$. Here the index 0 denotes the zero modes of the currents $J^{a}(z)=\sum_{n} J_{n}^{a} z^{-n-1}$. If both perturbations are present, the interaction preserves an $u(1 / 1)$ symmetry generated by

$$
H_{0}+\bar{H}_{0}, \quad J_{0}+\bar{J}_{0}, \quad G_{+0}+\bar{G}_{+0}, \quad \widehat{G}_{-0}+\overline{\widehat{G}}_{-0} .
$$

The perturbative study of the Dirac theory with a random potential and a random mass is very similar to the perturbative study of the random mass Ising model. However the crucial difference is that contrary to the randomness of the mass, the randomness of the potential is marginally relevant. At $g_{M}=0$, the one-loop beta function is given by $\dot{g}_{V}=8 g_{V}^{2}$. This means that $g_{V}$ grows at large distances. The infrared fixed point has not yet been determined [9]. Should the affine symmetry, present at the UV fixed point of the pure system, be restored at the IR point, then we believe this point should belong to the set we find in section 5 .

\section{The current algebra approach}

The critical free theory described by the action $S_{*}$ has a current algebra symmetry, $\operatorname{osp}(2 / 2)_{1}$, on both its holomorphic and antiholomorphic sectors. When randomness is introduced in the pure system the effective action is no longer critical, and the current algebra symmetry is reduced to a global $\operatorname{ssp}(2 / 2)$ symmetry. However at a new critical point the conformal invariance restores the current algebra symmetry. The value of the level $k$ is not preserved by the renormalization flow. Therefore its value at the IR fixed point could be different from its value at the UV point. It is possible to extract properties at the new critical point by studying the current algebra and its associated stress-energy tensor. In this context we consider $\operatorname{osp}(2 / 2)_{k}$ at arbitrary $k$ and obtain the Sugawara tensor.

\subsection{The $\operatorname{osp}(2 / 2)_{k}$ algebra}

In this section we write down the singular terms of the Operator Product Expansions [10] satisfied by the currents of the affine $\operatorname{osp}(2 / 2)$ algebra at level $k$. The non-trivial OPE of the currents are easily obtained from the currents (6). The level $k$ appearing below is equal to 1 for these currents. We find:

$$
\begin{array}{rlrlrl}
J(z) J(w) \sim \frac{k}{(z-w)^{2}} & ; & H(z) H(w) & \sim \frac{-k}{(z-w)^{2}} \\
J(z) G_{ \pm}(w) \sim \frac{ \pm 1}{z-w} G_{ \pm}(w) & ; & J(z) \widehat{G}_{ \pm}(w) & \sim \frac{ \pm 1}{z-w} \widehat{G}_{ \pm}(w) \\
H(z) G_{ \pm}(w) \sim \frac{1}{z-w} G_{ \pm}(w) & ; & H(z) \widehat{G}_{ \pm}(w) & \sim \frac{-1}{z-w} \widehat{G}_{ \pm}(w) \\
H(z) K(w) & \sim \frac{2}{z-w} K(w) & ; & H(z) \widehat{K}(w) & \sim \frac{-2}{z-w} \widehat{K}(w) \\
\widehat{G}_{ \pm}(z) G_{\mp}(w) & \sim & \frac{k}{(z-w)^{2}}+\frac{1}{z-w}(H(w) \pm J(w))
\end{array}
$$




$$
\begin{aligned}
\widehat{K}(z) K(w) & \sim \frac{2 k}{(z-w)^{2}}+\frac{4}{z-w} H(w) \\
G_{-}(z) G_{+}(w) \sim \frac{1}{z-w} K(w) & ; \quad \widehat{G}_{-}(z) \widehat{G}_{+}(w) \sim \frac{1}{z-w} \widehat{K}(w) \\
K(z) \widehat{G}_{ \pm}(w) \sim \frac{-2}{z-w} G_{ \pm}(w) & ; \quad \widehat{K}(z) G_{ \pm}(w) \sim \frac{2}{z-w} \widehat{G}_{ \pm}(w)
\end{aligned}
$$

Requiring the algebra to be associative constrains the possible central terms extensions to the ones appearing in the OPE's (14). We can rewrite these OPE's in a more compact form as

$$
J^{a}(z) J^{b}(w) \sim k \frac{\kappa^{a b}}{(z-w)^{2}}+f^{a b}{ }_{c} \frac{J^{c}(w)}{z-w} .
$$

The $f^{a b}{ }_{c}$ are the structure constants of the Lie superalgebra $o s p(2 / 2)$, and $\kappa^{a b}$ is proportional to its non-degenerate Killing form.

\subsection{The Sugawara stress-energy tensor}

We now construct the Sugawara stress-energy tensor [11]. It is bilinear in the currents defined in the previous section. Because of singularities which appear at coinciding points one has to consider a regularized version where normal ordered products of currents appear. This is equivalent to a point splitting procedure where the singular parts appearing in the OPE's of the currents are subtracted. We take the usual definition for the normal ordered product of two fields $A(z)$ and $B(w)$ :

$$
: A B:(w) \equiv \oint_{w} \frac{d z}{2 \pi i} \frac{A(z) B(w)}{z-w}
$$

It should be noted that this ordering prescription does not coincide in general with the Wick prescription used in (6). The Sugawara stress-energy tensor $T(z)$ is given by

$$
T(z)=\frac{1}{\kappa} \kappa_{a b}: J^{a}(z) J^{b}(z):
$$

where $\kappa_{a b}$ is the inverse of $\kappa^{a b}$. The constant $\kappa$ is determined by requiring $J^{a}(z)$ to be a primary field of conformal weight one:

$$
T(z) J^{a}(w) \sim \frac{J^{a}(w)}{(z-w)^{2}}+\frac{\partial J^{a}(w)}{z-w} .
$$

When calculating OPE's one has to add a minus sign each time two odd generators are permuted. We find

$$
\begin{aligned}
T(z) & =\frac{1}{4-2 k}\left(: H(z) H(z)-J(z) J(z)-\frac{1}{2}(K(z) \widehat{K}(z)+\widehat{K}(z) K(z))\right. \\
& \left.+\widehat{G}_{+}(z) G_{-}(z)-G_{-}(z) \widehat{G}_{+}(z)+\widehat{G}_{-}(z) G_{+}(z)-G_{+}(z) \widehat{G}_{-}(z):\right) .
\end{aligned}
$$

The Sugawara tensor also satisfies

$$
T(z) T(w) \sim \frac{c / 2}{(z-w)^{4}}+2 \frac{T(w)}{(z-w)^{2}}+\frac{\partial T(w)}{z-w} .
$$


For a general Lie superalgebra, it is easy to show that the Virasoro central charge $c$ is proportional to the superdimension; this is the difference between the number of even and odd generators. Therefore $c$ vanishes for the $\operatorname{sep}(2 / 2)$ algebra, and more generally for any superalgebra with an equal number of even and odd generators. More precisely one has:

$$
c=2 k \frac{\kappa_{a b} \kappa^{a b}}{\kappa}=2 k \frac{\operatorname{sdim} G}{\kappa}=0
$$

Because $c$ vanishes $T(z)$ is a primary field, contrary to the case $c \neq 0$ where $T$ is just a level-two descendant of the unit operator.

\section{Primary Fields}

We briefly describe the representations of $\operatorname{osp}(2 / 2)$ and find the corresponding conformal weights.

\subsection{Some $\operatorname{osp}(2 / 2)$ representations}

Unlike ordinary Lie algebras, the are two types of representations for most superalgebras. The typical representations are irreducible and are similar to those of ordinary Lie algebras. The atypical representations have no counterpart in the ordinary Lie algebra setting. They can be irreducible or not fully reducible (read reducible but indecomposable).

The superalgebra $\operatorname{osp}(2 / 2)$ is isomorphic to the superalgebra $\operatorname{spl}(2 / 1)$. The representation theory of the latter algebra was studied in [12, 13. The quadratic Casimir of $\operatorname{osp}(2 / 2)$ is

$$
C_{2}=\frac{1}{2}\left(H^{2}-\frac{1}{2}(K \widehat{K}+\widehat{K} K)-J^{2}+\widehat{G}_{+} G_{-}-G_{-} \widehat{G}_{+}+\widehat{G}_{-} G_{+}-G_{+} \widehat{G}_{-}\right) .
$$

The four even generators $K, \widehat{K}, H, J$ form a $s u(2) \oplus u(1)$ subalgebra. The correspondence with the notation of [12] is: $Q_{+}=-\widehat{K} / 2, Q_{-}=K / 2, Q_{3}=-H / 2, B=J / 2, V_{+}=$ $\widehat{G}_{+} / \sqrt{2}, V_{-}=-G_{+} / \sqrt{2}, W_{+}=-\widehat{G}_{-} / \sqrt{2}, W_{-}=G_{-} / \sqrt{2}$.

Let $b$ and $q_{3}$ be the eigenvalues of $B$ and $Q_{3}$. Generically, a representation $(b, q)$, $b \in \mathbb{C}, q=0, \frac{1}{2}, 1, \frac{3}{2}, \ldots$, contains four $s u(2) \oplus u(1)$ multiplets:

$$
\begin{aligned}
\left|b, q, q_{3}\right\rangle \quad, \quad q_{3} & =-q,-q+1, \ldots, q-1, q \text { if } q \geq 0, \\
\left|b+\frac{1}{2}, q-\frac{1}{2}, q_{3}\right\rangle \quad, \quad q_{3} & =-q+\frac{1}{2}, \ldots, q-\frac{3}{2}, q-\frac{1}{2} \text { if } q \geq \frac{1}{2}, \\
\left|b-\frac{1}{2}, q-\frac{1}{2}, q_{3}\right\rangle \quad, \quad q_{3} & =-q+\frac{1}{2}, \ldots, q-\frac{3}{2}, q-\frac{1}{2} \text { if } q \geq \frac{1}{2}, \\
\left|b, q-1, q_{3}\right\rangle \quad, \quad q_{3} & =-q+1, \ldots, q-2, q-1 \text { if } q \geq 1 .
\end{aligned}
$$

The action of the four even generators on these multiplets is the one implied by the notation. The four odd generators mix the different multiplets. The vector $|v\rangle=|b, q, q\rangle$ is a highest weight vector, i.e. it satisfies

$$
\widehat{K}|v\rangle=\widehat{G}_{+}|v\rangle=\widehat{G}_{-}|v\rangle=0 .
$$


The quadratic Casimir of this representation is $C_{2}=2\left(q^{2}-b^{2}\right)$.

If $b \neq \pm q$ the representation is denoted by $[b, q]$ and is typical; the quadratic and cubic Casimirs do not vanish. All the vectors in the representation can be obtained from the highest weight vector $|v\rangle$ by applying on it polynomials in the generators. The representation $[b, q]$ has dimension $8 q$. The representation $[0,1 / 2]$ is four-dimensional and contains one spin $1 / 2$ and two spin 0 multiplets.

\section{Atypical representations}

When $b= \pm q$ several kinds of atypical representations arise. Both Casimirs vanish, and yet these representations are not the trivial one-dimensional representation. One kind has dimension $4 q+1$ and is denoted by $[q]_{ \pm}$. To obtain $[q]_{+}$(resp. $[q]_{-}$) one drops the two multiplets (25) and (26) (resp. (24) and (26)). These representations are irreducible.

Atypical indecomposable representations

Generally, they are semidirect sums of atypical irreducible representations. They can contain two, three or four terms and they arise in tensor products of irreducible representations. An interesting example, containing four irreducible representations, arises in the tensor product of two representations $[0,1 / 2]$. The result is the direct sum of $[0,1]$ and an eight-dimensional representation which is the semidirect sum of $[1 / 2]_{-},[1 / 2]_{+}$and two $[0]$ representations. We call it $[0,-1 / 2,1 / 2,0]$ (see fig. $1 \mathrm{~b}$ ). The vector $s$ is invariant; it is annihilated by all the generators. The quadratic Casimir $C_{2}$ vanishes on all states except $t: C_{2} t=4 s$. We have

$$
\begin{aligned}
s & =\frac{1}{2}\left(\left|\frac{1}{2}, 0\right\rangle \otimes\left|-\frac{1}{2}, 0\right\rangle-\left|0, \frac{1}{2}\right\rangle \otimes\left|0,-\frac{1}{2}\right\rangle+\left|0,-\frac{1}{2}\right\rangle \otimes\left|0, \frac{1}{2}\right\rangle+\left|-\frac{1}{2}, 0\right\rangle \otimes\left|\frac{1}{2}, 0\right\rangle\right) \\
t & =\frac{1}{2}\left(\left|\frac{1}{2}, 0\right\rangle \otimes\left|-\frac{1}{2}, 0\right\rangle+\left|0, \frac{1}{2}\right\rangle \otimes\left|0,-\frac{1}{2}\right\rangle-\left|0,-\frac{1}{2}\right\rangle \otimes\left|0, \frac{1}{2}\right\rangle+\left|-\frac{1}{2}, 0\right\rangle \otimes\left|\frac{1}{2}, 0\right\rangle\right)
\end{aligned}
$$

\subsection{Tensor product of $\operatorname{osp}(2 / 2)$ representations}

The tensor product of two irreducible representations of a superalgebra is not necessarily completely reducible. Ref. [12] gives a sufficient condition for a tensor product of two $\operatorname{osp}(2 / 2)$ representations to be completely reducible, and the irreducible components. The result is the following

$$
\begin{gathered}
{[b, q] \otimes\left[b^{\prime}, q^{\prime}\right]=\left[b+b^{\prime}, q+q^{\prime}\right] \oplus\left[b+b^{\prime}, q+q^{\prime}-1\right] \oplus \ldots \oplus\left[b+b^{\prime},\left|q-q^{\prime}\right|\right]} \\
\oplus\left[b+b^{\prime}, q+q^{\prime}-1\right] \ldots \oplus\left[b+b^{\prime},\left|q-q^{\prime}\right|+1\right] \\
\oplus\left[b+b^{\prime}+1 / 2, q+q^{\prime}-1 / 2\right] \ldots \oplus\left[b+b^{\prime}+1 / 2,\left|q-q^{\prime}\right|+1 / 2\right] \\
\oplus\left[b+b^{\prime}-1 / 2, q+q^{\prime}-1 / 2\right] \ldots \oplus\left[b+b^{\prime}-1 / 2,\left|q-q^{\prime}\right|+1 / 2\right] \\
\text { if } \pm b>q \geq \frac{1}{2}, \quad \pm b^{\prime}>q^{\prime} \geq \frac{1}{2} .
\end{gathered}
$$

For the other values of $b, b^{\prime}$, this expression still gives the correct content of $s u(2) \oplus u(1)$ charges of the tensor product. This expression also permits to obtain some information on the reducibility: if two components have different Casimirs, then they belong to different (maybe indecomposable) representations. Vanishing Casimirs are, generally, signs of some pathologies. 


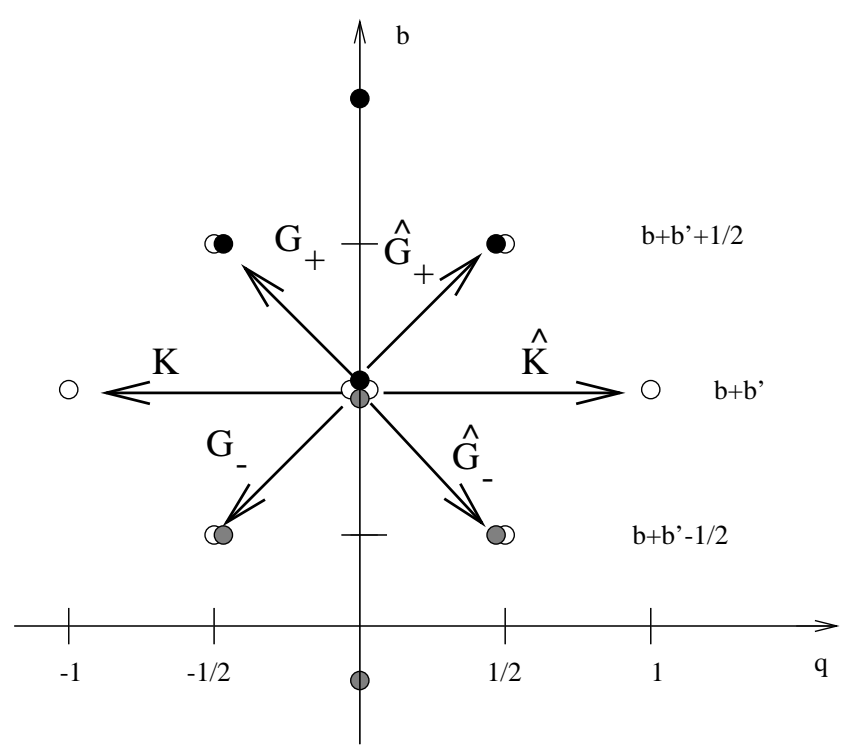

a)

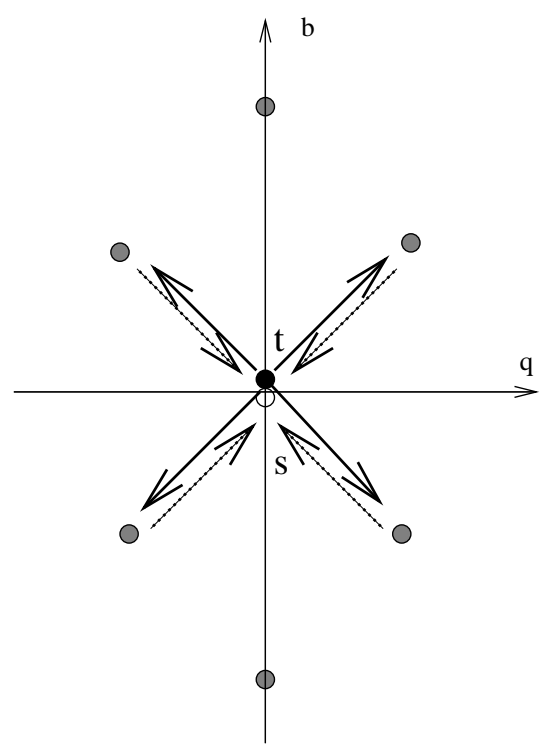

b)

Fig.1: a) The tensor product of the representations $[b, 1 / 2]$ and $\left[b^{\prime}, 1 / 2\right]$. If $b>1 / 2, b^{\prime}>$ $1 / 2$, the result is the direct sum of $\left[b+b^{\prime}, 1\right]$ (white dots), $\left[b+b^{\prime}+1 / 2,1 / 2\right]$ (black dots) and $\left[b+b^{\prime}-1 / 2,1 / 2\right]$ (grey dots). The arrows represent the action of the generators. b) The representation $[0,-1 / 2,1 / 2,0]$. The vector $t$ (black dot) is cyclic and $s$ (white dot) is invariant. The action of the fermionic generators $\widehat{G}_{+}, \widehat{G}_{-}, G_{+}$and $G_{-}$is represented by arrows.

\section{$4.3 \quad \operatorname{osp}(2 N / 2 N)$ representations}

When calculating the disorder average of product of correlation functions for the Ising and Dirac models, we can introduce an arbitrary number of copies of fermions and bosons. One is led is to consider $\operatorname{osp}(2 N / 2 N)$ as symmetry. The theory is consistent if the results of the calculations do not depend on $N$. One step in this direction is to show that the conformal dimensions of the primary fields are independent of $N$.

The main difference between the superalgebras $\operatorname{osp}(2 / 2)$ and $\operatorname{osp}(2 N / 2 N)$ with $N>1$ is that the even subalgebra of the first one is not simple. This is why representations of the former are indexed by a continuous parameter, while the representation of the latter are characterized only by discrete variables. According to the classification of the superalgebras [14], $\operatorname{osp}(2 / 2)$ is the first of the series $C(N+1)$, while $\operatorname{osp}(2 N / 2 N)$ is a superalgebra of the type $D(N, N)$.

The finite dimensional typical representation of the superalgebras were classified by Kac [14]. For $D(N, N)$, the typical representations are characterized by their highest weights, $\Lambda=\sum_{i=1}^{N} a_{i} \delta_{i}+\sum_{i=1}^{N} a_{N+i} \epsilon_{i} ; \delta_{i}$ and $\epsilon_{i}, i=1, N$ form an orthogonal basis with $\left(\epsilon_{i}, \epsilon_{j}\right)=-\delta_{i j},\left(\delta_{i}, \delta_{j}\right)=\delta_{i j},\left(\epsilon_{i}, \delta_{j}\right)=0$. The 'numerical marks' $a_{i}$ satisfy the following conditions:

i) $a_{i} \in \mathbb{Z}_{+}, i \neq N$

ii) $j=a_{N}-a_{N+1}-\ldots-a_{2 N-2}-\frac{1}{2}\left(a_{2 N-1}+a_{2 N}\right) \in \mathbb{Z}_{+}$,

iii) $a_{N+j+1}=\ldots=a_{2 N}=0$, if $j \leq N-2 ; a_{2 N-1}=a_{2 N}$, if $j=N-1$. 
The first two conditions express the fact that $\Lambda$ is a dominant weight for the even algebra $S p(2 N) \otimes O(2 N)$.

The value of the quadratic Casimir for the representation with highest weight $\Lambda$ is given, up to a normalization, by $(\Lambda, \Lambda+2 \rho)$, with $\rho=\rho_{0}-\rho_{1}$ and $\rho_{0(1)}$ is half the sum of the even (odd) positive roots. Considering the positive roots associated to the distinguished Dynkin diagram (the Dynkin diagram with a single fermionic root), we obtain

$$
\rho=\sum_{i=1}^{N}(N-i)\left(\epsilon_{i}-\delta_{N-i+1}\right) .
$$

We normalize the value of the quadratic Casimir of the adjoint representation $(2,0, \ldots, 0)$ to be 1 ; the Casimir of the representation $\left(a_{1}, \ldots, a_{2 N}\right)$ will be

$$
C_{2}(\Lambda)=\frac{1}{4} \sum_{i=1}^{N}\left[\left(a_{i}-i+1\right)^{2}-\left(a_{N+i}+N-i\right)^{2}\right]
$$

For the fundamental representation $(1,0, \ldots, 0)$ we have $C_{2}=1 / 4$, independent of $N$.

In order to obtain compatibility between the results obtained in the $\operatorname{osp}(2 / 2)$ and $\operatorname{osp}(2 N / 2 N)$ frameworks, the continous parameter $b$ of the $\operatorname{ssp}(2 / 2)$ representations has to be constrained to the discrete values compatible with the value of the discrete parameters of the $\operatorname{osp}(2 N / 2 N)$ representations.

\subsection{Conformal dimensions of the primary fields}

The primary fields $\phi^{\alpha}(w)$ are highest weight vectors of affine $\operatorname{osp}(2 / 2)_{k}$ and of the Virasoro algebra. In terms of operator products one has:

$$
\begin{aligned}
J^{a}(z) \phi^{\beta}(w) & \sim\left(T^{a}\right)^{\gamma \beta} \frac{\phi^{\gamma}(w)}{z-w}, \\
T(z) \phi^{\alpha}(w) & \sim \Delta \frac{\phi^{\alpha}(w)}{(z-w)^{2}}+\frac{\partial \phi^{\alpha}(w)}{(z-w)} .
\end{aligned}
$$

Using (15) and (29) one then shows that the matrices $T^{a}$ form a representation of the osp $(2 / 2)$ algebra. But this applies to any algebra. Using the Sugawara form (17) of the stress-energy tensor and (15) one obtains the conformal weights corresponding to a particular representation. For the representations $[b, q]$ and $[q]_{ \pm}$the Casimir $C_{2}$ is diagonal and we obtain:

$$
\Delta_{(b, q)}=\frac{1}{\kappa} \kappa_{a b} T^{a} T^{b}=\frac{2\left(q^{2}-b^{2}\right)}{2-k} .
$$

We are also able to calculate the conformal dimensions of the primary fields for all $N$. Due to the fact that the dual Coxeter number of $\operatorname{osp}(2 N / 2 N)$ equals 1 for all $N$, these conformal dimensions do not change if we change the number of copies to $N^{\prime}>N$; for example

$$
\Delta_{(1,0, \ldots, 0)}=\frac{1}{4-2 k}
$$

is the conformal dimension of the fields in the fundamental representation.

The conformal dimensions are potentially negative; this reflects the non-unitarity of the theory we considered. 


\section{Local Operators and Quantization of $k$}

As seen in section two the interactions generated by the random mass and random scalar potential conserve diagonal $\operatorname{osp}(2 / 2), J_{x}^{a}=J_{0}^{a} \pm \bar{J}_{0}^{a}$. We expect to find in the spectrum of the theory, at the new fixed point for the potential interaction, particles which carry the same charges $H_{x}$ and $J_{x}$ as the original ones (bosons and fermions). The fields corresponding to these particles are no more holomorphic or antiholomorphic, but they can be made up of the new primary fields. Suppose that they are of the form

$$
\phi(z, \bar{z})=\phi_{[b, q]}(z) \phi_{\left[b^{\prime}, q^{\prime}\right]}(\bar{z})
$$

where $\phi_{[b, q]}(z)\left(\phi_{\left[b^{\prime}, q^{\prime}\right]}(\bar{z})\right)$ are primary fields belonging to the representation $[b, q]$, respectively $\left[b^{\prime}, q^{\prime}\right]$ of the two chiral algebras $J^{a}(z)$ and $\bar{J}^{a}(\bar{z})$.

The charges of the bosons and fermions with respect to the currents $J_{x}^{a}$ are:

$\begin{array}{rrr} & J_{x} & H_{x} \\ \psi_{+}(z) & 1 / 2 & 0 \\ \psi_{-}(z) & -1 / 2 & 0 \\ \gamma(z) & 0 & 1 / 2 \\ \eta(z) & 0 & -1 / 2\end{array}$

We have to identify the representation $[0,1 / 2]$ in the tensor product of the representations $[b, q]$ and $\left[b^{\prime}, q^{\prime}\right]$. Using equation (28) we obtain the following possibilities

$$
\text { a) } \left.q=q^{\prime}, \quad b+b^{\prime} \pm 1 / 2=0, \quad b\right) \quad\left|q-q^{\prime}\right|=1 / 2, \quad b+b^{\prime}=0 .
$$

As in [2], in order to obtain a sensible theory, we impose the constraint of locality on the fields $\phi(z, \bar{z})$. The OPE of two such composite fields can be written as

$$
\mathcal{O}_{a}(z, \bar{z}) \mathcal{O}_{b}(0,0)=\sum_{c} C_{a b}^{c} z^{\Delta_{c}-\Delta_{a}-\Delta_{b}} \bar{z}^{\bar{\Delta}_{c}-\bar{\Delta}_{a}-\bar{\Delta}_{b}} \mathcal{O}_{c}(0,0)
$$

The operators $\mathcal{O}_{a}$ and $\mathcal{O}_{b}$ are said to be mutually local if the OPE is single-valued, which means that the spin difference is an integer

$$
s_{c}-s_{a}-s_{b}=\Delta_{c}-\Delta_{a}-\Delta_{b}-\bar{\Delta}_{c}+\bar{\Delta}_{a}+\bar{\Delta}_{b} \in \mathbb{Z} .
$$

Let us take $\mathcal{O}_{a}(z, \bar{z})=\mathcal{O}_{b}(z, \bar{z})=\phi_{[b, q]}(z) \phi_{\left[b^{\prime}, q^{\prime}\right]}(\bar{z})$. We evaluate the left and right chirality OPE's separately. The operators appearing in the OPE for the left chirality are the operators associated to the representations $\alpha$ appearing in the tensor product $[b, q] \otimes[b, q]$ (see eq. (28))

$$
\phi_{[b, q]}(z) \phi_{[b, q]}(0) \sim \sum_{\alpha} z^{\Delta_{\alpha}-2 \Delta_{[b, q]}} \phi_{\alpha}(0)
$$

and similarly for the right chirality, with $[b, q]$ replaced by $\left[b^{\prime}, q^{\prime}\right]$. We ignored the possible existence of logarithms in this OPE (when two conformal dimensions are degenerate). We also supposed that all the primary fields allowed by the $\operatorname{osp}(2 / 2)$ selection rules (and their descendants) appear in the OPE. For a Kac-Moody algebra this is not always the case; the operator content of the theory depends on the value of the level $k$. In fact the 
calculation shows that the constraint of locality depends very weakly on how many of the operators $\phi_{\alpha}$ really appear in the OPE.

The condition $s_{c}-s_{a}-s_{b} \in \mathbb{Z}$ is fulfilled for all the possible operators $\mathcal{O}_{c}(0,0)$ in (35) if

$$
\begin{gathered}
2 l(4 q-1) \in \mathbb{Z}, \quad 2 l(4 q-2) \in \mathbb{Z}, \quad 4 l(q-b) \in \mathbb{Z}, \\
2 l\left(4 q^{\prime}-1\right) \in \mathbb{Z}, \quad 2 l\left(4 q^{\prime}-2\right) \in \mathbb{Z}, \quad 4 l\left(q^{\prime}-b^{\prime}\right) \in \mathbb{Z}, \\
\text { and } \quad 4 l\left(q^{2}-b^{2}-q^{\prime 2}+b^{\prime 2}\right) \in \mathbb{Z},
\end{gathered}
$$

where $l=1 /(2-k)$. With the two possibilities in (34) we obtain the following restriction on $k$ :

$$
k=2-\frac{1}{l}, \quad l \in \mathbb{Z} .
$$

\section{Logarithmic operators}

We argue that the appearance of logarithmic operators is a general feature of theories with an underlying superalgebra symmetry, as long as indecomposable representations are concerned. We give as an example the occurrence of logarithmic correlations for $\operatorname{osp}(2 / 2)$.

The first logarithmic correlation functions in conformal field theory were obtained by Rozansky and Saleur in [15] in the framework of the $G L(1,1)$ WZW model. Later Gurarie [16] pointed out that the logarithms are related to non-diagonalizable Virasoro generators. See also [1] for disordered models, [17], and [18] in relation to the fractional quantum Hall effect. Suppose we are given two operators $A_{1}(z), A_{2}(z)$ with:

$$
\begin{aligned}
T(z) A_{1}(0) & \sim \frac{\Delta}{z^{2}} A_{1}(0)+\frac{\partial A_{1}(0)}{z} \\
T(z) A_{2}(0) & \sim \frac{\Delta}{z^{2}} A_{2}(0)+\frac{\partial A_{2}(0)}{z}+\frac{a}{z^{2}} A_{1}(0) .
\end{aligned}
$$

Then they transform under the dilatations $z^{\prime}=\alpha z$ as

$$
A_{1}^{\prime}\left(z^{\prime}\right)=\alpha^{-\Delta} A_{1}(z), \quad A_{2}^{\prime}\left(z^{\prime}\right)=\alpha^{-\Delta} A_{2}(z)+a \log \alpha A_{1}(z) .
$$

And more generally one has:

$$
\begin{gathered}
{\left[L_{n}, A_{1}(z)\right]=\left(z^{n+1} \partial_{z}+\Delta(n+1) z^{n}\right) A_{1}(z)} \\
{\left[L_{n}, A_{2}(z)\right]=\left(z^{n+1} \partial_{z}+\Delta(n+1) z^{n}\right) A_{2}(z)+a(n+1) z^{n} A_{1}(z) .}
\end{gathered}
$$

This gives rise to differential equations for the correlation functions of the operators $A_{1}(z)$ and $A_{2}(z)$, through the conformal Ward identities for $n=-1,0,1$. Let us determine the two-point functions of these operators. The $n=0$ differential equations for these correlation functions are

$$
\begin{gathered}
\left(z \partial_{z}+2 \Delta\right)\left\langle A_{1}(z) A_{1}(0)\right\rangle=0, \quad\left(z \partial_{z}+2 \Delta\right)\left\langle A_{2}(z) A_{1}(0)\right\rangle+a\left\langle A_{1}(z) A_{1}(0)\right\rangle=0 \\
\left(z \partial_{z}+2 \Delta\right)\left\langle A_{2}(z) A_{2}(0)\right\rangle+a\left\langle A_{1}(z) A_{2}(0)\right\rangle+a\left\langle A_{2}(z) A_{1}(0)\right\rangle=0
\end{gathered}
$$


while for $n=1$ we have

$$
\begin{aligned}
& \left(z \partial_{z}+2 \Delta\right)\left\langle A_{2}(z) A_{1}(0)\right\rangle+2 a\left\langle A_{1}(z) A_{1}(0)\right\rangle=0 \\
& \left(z \partial_{z}+2 \Delta\right)\left\langle A_{2}(z) A_{2}(0)\right\rangle+2 a\left\langle A_{1}(z) A_{2}(0)\right\rangle=0 .
\end{aligned}
$$

This implies

$$
\begin{gathered}
\left\langle A_{1}(z) A_{1}(0)\right\rangle=0,\left\langle A_{2}(z) A_{1}(0)\right\rangle=\left\langle A_{1}(z) A_{2}(0)\right\rangle=C z^{-2 \Delta} \\
\left\langle A_{2}(z) A_{2}(0)\right\rangle=\left(-2 a C \log z+C^{\prime}\right) z^{-2 \Delta}
\end{gathered}
$$

with $C, C^{\prime}$ arbitrary constants. This system of correlation functions appeared in [16, 11. Thus if the operators $A_{1}, A_{2}$ satisfy the OPE (41 42) they can have logarithmic correlators, and $A_{2}(z)$ is the logarithmic operator related to $A_{1}(z)$.

In the following we give an example of such operators appearing in the indecomposable representations of the $\operatorname{osp}(2 / 2)$ superalgebra. Consider fields $\phi_{i}(z)$ in the atypical indecomposable representation $[0,-1 / 2,1 / 2,0]$ (fig. 1b). The structure of the generators $T^{a}$ is

$$
T^{a}=\begin{gathered}
\lambda \\
\lambda \\
\lambda^{\prime} \\
s \\
t
\end{gathered}\left(\begin{array}{cccc}
t^{a} & 0 & 0 & * \\
0 & t^{\prime a} & 0 & * \\
* & * & 0 & 0 \\
0 & 0 & 0 & 0
\end{array}\right)
$$

where $\lambda=[1 / 2]_{+}, \lambda^{\prime}=[1 / 2]_{-}$and the stars stand for possible non-zero matrix elements. The action of the currents on the fields takes the form

$$
J^{a}(z) \phi_{1}(0)=T_{1}^{a} \frac{\phi_{1}(0)}{z}, \quad J^{a}(z) \phi_{2}(0)=T_{1}^{a} \frac{\phi_{2}(0)}{z}+T_{2}^{a} \frac{\phi_{1}(0)}{z},
$$

where the index 1 refers to the invariant subspace. This form is typical of indecomposable representations of any algebra. We find the most singular term arising from the $J-\phi$ contractions in $\left\langle J^{a}(z) J^{b}(w) \phi_{2}(0) \prod \mathcal{O}\right\rangle$. Using the definition

$$
T(z)=\lim _{z \rightarrow w} \frac{1}{\kappa}\left(\kappa_{a b} J^{a}(z) J^{b}(w)-k \frac{\kappa_{a b} \kappa^{a b}}{(z-w)^{2}}\right)
$$

we conclude that

$$
\begin{aligned}
T(z) \phi_{1}(0) & =\frac{\Delta}{z^{2}} \phi_{1}(0)+\frac{1}{z} \partial \phi_{1}(0) \\
T(z) \phi_{2}(0) & =\frac{\Delta}{z^{2}} \phi_{2}(0)+\frac{1}{\kappa} \frac{\kappa_{a b}\left(T_{1}^{a} T_{2}^{b}+T_{2}^{a} T_{1}^{b}\right)}{z^{2}} \phi_{1}(0)+\frac{1}{z} \partial \phi_{2}(0),
\end{aligned}
$$

where $\Delta=\frac{1}{\kappa} \kappa_{a b} T_{1}^{a} T_{1}^{b}$. This non-diagonal action of the Virasoro algebra is the direct consequence of the existence of an indecomposable representation at the affine level. For the representation at hand one gets $C_{2} t \sim s, C_{2} s=0$, and for the fields $\phi_{s}$ and $\phi_{t}$ with zero $\operatorname{osp}(2 / 2)$ charges:

$$
T(z) \phi_{s}(0) \sim \frac{\partial \phi_{s}(0)}{z}, \quad T(z) \phi_{t}(0) \sim a \frac{\phi_{s}(0)}{z^{2}}+\frac{\partial \phi_{t}(0)}{z} .
$$


According to the previous discussion their correlation functions are

$$
\left\langle\phi_{s}(z) \phi_{s}(0)\right\rangle=0,\left\langle\phi_{t}(z) \phi_{s}(0)\right\rangle=C,\left\langle\phi_{t}(z) \phi_{t}(0)\right\rangle=-2 a C \log z+C^{\prime} .
$$

We can also solve the differential equations for $\left\langle\phi_{s}\right\rangle$ and $\left\langle\phi_{t}\right\rangle$ to find $\left\langle\phi_{s}\right\rangle=0,\left\langle\phi_{t}\right\rangle=c$.

The indecomposable representation we considered appear in the tensor product of two fundamental representations. Thus logarithmic terms are expected to appear in the four point functions (if the logarithmic operators are not identically zero, as seems to be the case for the free bosons/fermions $k=1$ ).

\section{Four-point functions}

We now calculate a set of four-point correlation functions for primary fields in the $\left[0, \frac{1}{2}\right]$ representation of $\operatorname{ssp}(2 / 2)$. As explained in section 6 we expect to find a logarithmic dependence.

\subsection{Differential equations}

Consider the basis

$$
|1\rangle=\left|0, \frac{1}{2}\right\rangle,|2\rangle=\left|-\frac{1}{2}, 0\right\rangle,|3\rangle=\left|\frac{1}{2}, 0\right\rangle,|4\rangle=\left|0,-\frac{1}{2}\right\rangle,
$$

where $|1\rangle$ and $|4\rangle$ are even (bosonic) and $|2\rangle$ and $|3\rangle$ are odd (fermionic). These vectors are labeled by the eigenvalues of $B$ and $Q_{3}$. The odd generators are given by:

$$
\begin{gathered}
V_{+}=\sqrt{2}\left(\begin{array}{llll}
0 & \epsilon & 0 & 0 \\
0 & 0 & 0 & 0 \\
0 & 0 & 0 & \alpha \\
0 & 0 & 0 & 0
\end{array}\right) \quad, \quad W_{-}=\sqrt{2}\left(\begin{array}{cccc}
0 & 0 & 0 & 0 \\
-\beta & 0 & 0 & 0 \\
0 & 0 & 0 & 0 \\
0 & 0 & \gamma & 0
\end{array}\right) \\
W_{+}=\sqrt{2}\left(\begin{array}{llll}
0 & 0 & \gamma & 0 \\
0 & 0 & 0 & \beta \\
0 & 0 & 0 & 0
\end{array}\right) \quad, \quad V_{-}=\sqrt{2}\left(\begin{array}{cccc}
0 & 0 & 0 & 0 \\
0 & 0 & 0 & 0 \\
-\alpha & 0 & 0 & 0 \\
0 & \epsilon & 0 & 0
\end{array}\right)
\end{gathered}
$$

The four parameters appearing in (53 54) are constrained by

$$
4 \alpha \gamma=1, \quad 4 \beta \epsilon=1 \text {. }
$$

Thus there are two free parameters which correspond to arbitrary relative normalizations of the $s u(2)$ doublet $(|1\rangle,|4\rangle)$, and the two singlets $|2\rangle$ and $|3\rangle$.

The vacuum invariance under the zero modes of the affine algebra, or equivalently invariance under global gauge transformations, imply the following linear equations

$$
\sum_{i=1}^{4} T_{i}^{a}\left\langle\phi_{1}\left(z_{1}\right) \phi_{2}\left(z_{2}\right) \phi_{3}\left(z_{3}\right) \phi_{4}\left(z_{4}\right)\right\rangle=0 \text {. }
$$

It is understood that minus signs arise each time we permute two fermionic objects, and omit the antiholomorphic dependence and indices of the fields. Similar equations hold for 
the 'right' (antiholomorphic) chiral generators. These equations are solved in terms of invariant tensors for the representations at hand. We have found the explicit expressions for three linearly independent tensors which we give in the appendix.

Requiring the fields of a specific module to be both affine and Virasoro primary gives a set of equations relating the fields. Such equations give partial differential equations, the Knizhnik-Zamolodchikov equations [19], for the correlation functions. One gets

$$
\left[\frac{1}{2} \frac{\partial}{\partial z_{1}}-\frac{\kappa_{a b}}{\kappa} \sum_{j=2}^{4} \frac{1}{z_{1}-z_{j}} T_{1}^{a} T_{j}^{b}\right]\left\langle\phi_{1}\left(z_{1}\right) \prod_{j=2}^{4} \phi_{j}\left(z_{j}\right)\right\rangle=0 .
$$

After a conformal transformation this equation reduces to a matrix differential equation for the correlators

$$
F^{i_{1} i_{2} i_{3} i_{4}}(z)=\lim _{w \rightarrow \infty} w^{2 \Delta_{4}}\left\langle\phi^{i_{1}}(z) \phi^{i_{2}}(0) \phi^{i_{3}}(1) \phi^{i_{4}}(w)\right\rangle .
$$

One gets

$$
\frac{1}{2} \partial F(z)=\frac{1}{\kappa}\left(\frac{1}{z} \mathcal{P}+\frac{1}{z-1} \mathcal{Q}\right) F(z)
$$

where

$$
z=\frac{z_{12} z_{34}}{z_{23} z_{41}}, \mathcal{P}=\kappa_{a b} T_{1}^{a} \otimes T_{2}^{b}, \quad \mathcal{Q}=\kappa_{a b} T_{1}^{a} \otimes T_{3}^{b} .
$$

Similar equations hold for the antiholomorphic sector. The complete correlator is given by

$$
\mathcal{F}\left(z_{1}, z_{2}, z_{3}, z_{4}\right)=z_{14}^{-2 \Delta_{1}} z_{23}^{-\Delta_{1}-\Delta_{2}-\Delta_{3}+\Delta_{4}} z_{24}^{\Delta_{1}-\Delta_{2}+\Delta_{3}-\Delta_{4}} z_{34}^{\Delta_{1}+\Delta_{2}-\Delta_{3}-\Delta_{4}} F(z) .
$$

For the foregoing representations $\Delta_{i}=1 /(2 x)$ and the $z_{i j}$ prefactor reduces to $z_{14}^{-x^{-1}} z_{23}^{-x^{-1}}$, where

$$
x=2-k .
$$

The correlators have the $\operatorname{osp}(2 / 2) \times \operatorname{osp}(2 / 2)$ invariant decomposition

$$
F^{i_{1} i_{2} i_{3} i_{4}, j_{1} j_{2} j_{3} j_{4}}(z, \bar{z})=\sum_{A, B=1}^{3} I_{A}^{i_{1} i_{2} i_{3} i_{4}} \bar{I}_{B}^{j_{1} j_{2} j_{3} j_{4}} F_{A B}(z, \bar{z}) .
$$

The tensors are given in the appendix and the nine scalar functions satisfy the differential equations:

$$
x \frac{\partial F}{\partial z}=\left[\frac{1}{z} P+\frac{1}{z-1} Q\right] F,
$$

where $F$ now denotes the vector $\left(F_{1 B}, F_{2 B}, F_{3 B}\right)$ for all $B$. There are similar equations for the antiholomorphic dependence, for the vector $\left(F_{A 1}, F_{A 2}, F_{A 3}\right)$. We give the matrices $P$ and $Q$ in the appendix. Let $f_{A}(z)=F_{A B}(z)$ for any given $B$. It is straightforward to reduce this first-order matrix differential equation to the following set of equations:[

$$
\begin{aligned}
& x^{3} z^{3}(1-z)^{3} \partial^{3} f_{3}(z)+x^{2}(1+2 x) z^{2}(1-z)^{2}(1-2 z) \partial^{2} f_{3}(z)+x z(1-z)[-1-x \\
& +2 x z-2 x(2+x) z(1-z)] \partial f_{3}(z)+(-x-1+2 z+2 x z(1-z)) f_{3}(z)=0
\end{aligned}
$$

\footnotetext{
${ }^{1}$ It is amusing to note that the third-order differential equations for $f_{1}(z)$ and $f_{2}(z)$ reveal two apparent singularities at $z=2$ and $z=\frac{1}{2}$.
} 


$$
\begin{aligned}
& f_{2}(z)=-\frac{1}{4 \epsilon \gamma x z(1-z)}\left(x^{2} D^{2} f_{3}(z)+2 x(1-z) D f_{3}(z)+(1-2 z) f_{3}(z)\right) \\
& f_{1}(z)=\frac{1}{4 \epsilon \gamma}\left(x D f_{3}(z)-f_{3}(z)\right)+(z-2) f_{2}(z)
\end{aligned}
$$

where $D=z(1-z) \partial$. As expected the equation for $f_{3}$ is fuchsian with three regular singularities at 0,1 and $\infty$. The corresponding indices are $\left(-x^{-1},-x^{-1}, 1+x^{-1}\right)$, $\left(-x^{-1}, 1-x^{-1}, x^{-1}\right)$ and $\left(0,0,1+2 x^{-1}\right)$. The degeneracy of some indices, i.e. when the difference of two indices belongs to $\mathbb{Z}$, signals the eventual presence of logarithmic solutions. Thus one is naturally lead to distinguish between different values of $x$ 's.

We were able to express the functions $f_{i}$ in terms of generalized hypergeometric functions. We obtained:

$$
\begin{gathered}
F^{1423}(z)=f_{3}(z)=\frac{1}{2}(1-x D) F^{-}(z), \\
x^{3} z^{3}(1-z)^{3} \partial^{3} F^{-}+x^{2}(3 x+1) z^{2}(1-z)^{2}(1-2 z) \partial^{2} F^{-} \\
+z(1-z)\left(x^{2}(x+1)(1-6 z(1-z))-x\right) \partial F^{-}-(1-2 z) F^{-}=0 .
\end{gathered}
$$

Equation (67) is fuchsian with three regular singular points at 0,1 and $\infty$. The indices at 0 and 1 are $\left(-\frac{1}{x},-\frac{1}{x}, \frac{1}{x}\right)$. The solutions of equation (67) can be written as generalized hypergeometric functions:

$$
\begin{aligned}
& F_{0}^{-}(z)=(z(1-z))^{-\frac{1}{x}}{ }_{3} F_{2}\left(\frac{1}{2},-\frac{1}{x}, 1-\frac{1}{x} ; 1,-\frac{2}{x} ; 4 z(1-z)\right) \\
& F_{1}^{-}(z)=F_{0}^{-}(z) \log [4 z(1-z)]+(z(1-z))^{-\frac{1}{x}} \sum_{n \geq 0} c_{n}(4 z(1-z))^{n} \\
& F_{2}^{-}(z)=(z(1-z))^{\frac{1}{x}}{ }_{3} F_{2}\left(\frac{1}{x}, 1+\frac{1}{x}, \frac{1}{2}+\frac{2}{x} ; 1+\frac{2}{x}, 1+\frac{2}{x} ; 4 z(1-z)\right)
\end{aligned}
$$

where

$$
\begin{gathered}
{ }_{3} F_{2}\left(a_{1}, a_{2}, a_{3} ; b_{1}, b_{2} ; z\right) \equiv \sum_{n \geq 0} \frac{\left(a_{1}\right)_{n}\left(a_{2}\right)_{n}\left(a_{3}\right)_{n}}{\left(b_{1}\right)_{n}\left(b_{2}\right)_{n} n !} z^{n}, \quad(a)_{n}=a(a+1) \cdots(a+n-1), \\
c_{n}=\frac{d}{d s}\left(\frac{\Gamma\left(n+s+\frac{1}{2}\right) \Gamma\left(n+s-\frac{1}{x}\right) \Gamma\left(n+s+1-\frac{1}{x}\right) \Gamma(s+1)^{2} \Gamma\left(s+1-\frac{2}{x}\right)}{\Gamma(n+s+1)^{2} \Gamma\left(n+s+1-\frac{2}{x}\right) \Gamma\left(s+\frac{1}{2}\right) \Gamma\left(s-\frac{1}{x}\right) \Gamma\left(s+1-\frac{1}{x}\right)}\right)_{s=0} .
\end{gathered}
$$

It must be noted that although the solutions look symmetric under the exchange $z \leftrightarrow 1-z$ it is not possible to have three linearly independent solutions with such a symmetry. When the series defining the hypergeometric functions do not truncate there are singularities at $u=4 z(1-z)=1$ of the type $\log (1+\sqrt{1-u})$. And since the reciprocal image of the interior of the $u$-unit disc consists of two disjoint lobes, in the $z$ plane, centered around 0 and 1, the change $z \rightarrow 1-z$ changes the determination of the hypergeometric function around $z=1$ from what it is around $z=0$. This feature can be seen explicitly for the cases $x=1$ and $x=1 / 2$ below.

Before analyzing some specific solutions let us note that after the change of function $F^{-}(z)=(z(1-z))^{-\frac{1}{x}} G(z)$ one obtains

$$
\begin{gathered}
z^{2}(z-1)^{2} \partial^{3} G+z(1-z)\left(K_{1} z+K_{2}(z-1)\right) \partial^{2} G+\left(L_{1} z^{2}+L_{2}(z-1)^{2}\right. \\
\left.+L_{3} z(z-1)\right) \partial G+\left(M_{1} z+M_{2}(z-1)\right) G=0,
\end{gathered}
$$

\footnotetext{
${ }^{2}$ See $[20$ or any book on differential equations.
} 
where

$$
K_{1}=K_{2}=3-\frac{2}{x}, L_{1}=L_{2}=1-\frac{2}{x}, \quad L_{3}=4\left(1-\frac{1}{x}\right)^{2}, \quad M_{1}=M_{2}=\frac{2}{x}\left(\frac{1}{x}-1\right) .
$$

Equations of this type appeared for correlations functions containing a level three null vector in minimal models (see ref. [21]). There are integral representations for the solutions

$$
G(z)=\int_{C_{1}} d t_{1} \int_{C_{2}} d t_{2} t_{1}^{\frac{1}{x}-1}\left(t_{1}-1\right)^{\frac{1}{x}-1}\left(t_{1}-z\right)^{\frac{1}{x}} t_{2}^{\frac{1}{x}-1}\left(t_{2}-1\right)^{\frac{1}{x}-1}\left(t_{2}-z\right)^{\frac{1}{x}}\left(t_{1}-t_{2}\right)^{-\frac{4}{x}} .
$$

These integrals can however diverge, depending on the contours $C_{1}$ and $C_{2}$ and the value of $x$.

\subsection{The level 1 correlators}

For $x=1$, i.e. $k=1$, we are able to write the solutions of (67) in a closed form:

$$
F^{-}(z)=g_{0} \frac{1}{z(1-z)}+g_{1}\left(1+\frac{\log z}{z(1-z)}\right)+g_{2}\left(1+\frac{\log (1-z)}{z(1-z)}\right) .
$$

We then want to construct the physical correlation functions which must be single-valued on the whole Riemann sphere. It is therefore enough to ensure this property at the two singular points 0 and 1 . It is easy to see that one can only have

$$
\begin{aligned}
F_{A B}(z, \bar{z}) & =\alpha_{0} F_{A}^{(0)}(z) F_{B}^{(0)}(\bar{z})+\alpha_{1}\left(F_{A}^{(0)}(z) F_{B}^{(1)}(\bar{z})+F_{A}^{(1)}(z) F_{B}^{(0)}(\bar{z})\right) \\
& +\alpha_{2}\left(F_{A}^{(0)}(z) F_{B}^{(2)}(\bar{z})+F_{A}^{(2)}(z) F_{B}^{(0)}(\bar{z})\right)
\end{aligned}
$$

where the constants $\alpha_{i}$ are independent of the indices $A, B$, and

$$
\begin{gathered}
F_{1}^{(0)}(z)=\frac{z-2}{4 \epsilon \gamma(1-z)}, F_{2}^{(0)}(z)=\frac{2 z-1}{4 \epsilon \gamma z(1-z)}, F_{3}^{(0)}(z)=\frac{1}{z} \\
F_{1}^{(1)}(z)=\frac{1}{4 \epsilon \gamma}\left(-1+\frac{z-2}{1-z} \log z\right), F_{2}^{(1)}(z)=\frac{1}{4 \epsilon \gamma z(1-z)}(1-z+(2 z-1) \log z), \\
F_{3}^{(1)}(z)=\frac{1}{2 z}(z-1+2 \log z), F_{1}^{(2)}(z)=\frac{1}{4 \epsilon \gamma}\left(\frac{z}{z-1}+\frac{2-z}{1-z} \log (1-z)\right), \\
F_{2}^{(2)}(z)=\frac{1}{4 \epsilon \gamma z(1-z)}(z+(1-2 z) \log (1-z)), F_{3}^{(2)}(z)=\frac{1}{2 z}\left(\frac{z(z-2)}{1-z}-2 \log (1-z)\right)
\end{gathered}
$$

This form of $F_{A B}$ differs from the usual diagonal one one finds for minimal models. Here there are three so far unconstrained constants (one constant is an overall normalization), even after requiring monodromy invariance around the three singular points.

We still have to require crossing symmetry among the correlators:

$$
F^{i j k l}(z, \bar{z})=F^{i k j l}(1-z, 1-\bar{z}), \quad F^{i j k l}(z, \bar{z})=z^{-1 / x} \bar{z}^{-1 / x} F^{i l k j}(1 / z, 1 / \bar{z}) .
$$

These conditions imply that $\alpha_{1}=\alpha_{2}=0$. These correlators are thus free from logarithms.

At level one the free fields $\left(\gamma, \psi_{-}, \psi_{+}, \eta\right)$ provide a representation $\left[0, \frac{1}{2}\right]$ of the affine algebra $\operatorname{osp}(2 / 2)_{1}$. The corresponding correlation functions are then easily calculated by making use of Wick's theorem. They agree with the results obtained from the above calculation, and are free of logarithms. 
We then looked at $x=1 / 2(k=3 / 2)$ correlators for which we explicitly found the functions $F_{A}^{(i)}$. Again the physical correlators were free from logarithms. It is possible to show that such is the case for all non-vanishing integer values of $x^{-1}$. The solutions $F_{A}^{(i)}$ have the same form as those for $x=1$ and $x=1 / 2$, namely a combination of rational functions of $z$ and of $\log z$ and $\log (1-z)$. We were not able to determine the connection matrices between functions with arguments $z, 1-z$ and $1 / z$. The limiting values for those obtained in [21] depend on the way the limit is taken. But it is enough to know the form of these matrices to implement the crossing symmetries. In doing so we also used the matrices connecting the tensors $I_{a}$ with transposed indices, and which can be found in the appendix. We found that $\alpha_{1}=\alpha_{2}=0$. Note that the values of $k$ considered here are exactly those of equation (40). We comment on this result in the conclusion.

From the four-point correlation functions we can obtain information about the fusion rules for the $[0,1 / 2]$ representation. As the contribution from the conformal blocks 1 and 2 vanishes we infer that the corresponding operators do not appear in the OPE $\phi_{[0,1 / 2]}(z) \phi_{[0,1 / 2]}(w)$. The only primary field appearing in their OPE corresponds to the operator $\phi_{t}$, which is now the identity.

\subsection{Logarithms for generic $x$}

We now consider the generic case where $2 x^{-1}$ is not an integer. Contrary to what happens in the preceding section, it does not seem possible to isolate a solution which is regular at both $z=0$ and $z=1$. A solution regular at 0 will have a logarithmic singularity at $z=1$ and vice-versa. This means that there is no way to get rid of logarithms if the correlators are not vanishing. We expect that there should be logarithmic operators, and the knowledge of the four-point functions allows one to find the OPE's of the fields we consider, including the logarithmic ones.

\subsection{Squared logarithms}

We now turn to the third and last possible scenario where $2 x^{-1}$ is an integer but $x^{-1}$ is not an integer: $x^{-1}=n+1 / 2, n \in \mathbb{Z}$. The theory of fuchsian differential equations indicates that there might be a solution with a $\log ^{2} z$ dependence. A careful analysis shows that this is indeed the case. And again it seems impossible to isolate a logarithm-free solution. However one has to modify the form of $F_{A B}$. Write the solutions as

$$
\begin{gathered}
F_{A}^{(0)}(z)=F_{A}(z), \quad F_{A}^{(1)}(z)=F_{A}(z) \log z+H_{A}(z), \\
F_{A}^{(2)}(z)=F_{A}(z) \log ^{2} z+2 H_{A}(z) \log z+G_{A}(z) .
\end{gathered}
$$

Then the monodromy invariant combinations around $z=0$ are

$$
\begin{aligned}
F_{A B}(z, \bar{z}) & =\alpha_{0} F_{A}^{(0)}(z) F_{B}^{(0)}(\bar{z})+\alpha_{1}\left(F_{A}^{(0)}(z) F_{B}^{(1)}(\bar{z})+F_{A}^{(1)}(z) F_{B}^{(0)}(\bar{z})\right) \\
& +\alpha_{2}\left(F_{A}^{(0)}(z) F_{B}^{(2)}(\bar{z})+F_{A}^{(2)}(z) F_{B}^{(0)}(\bar{z})+2 F_{A}^{(1)}(z) F_{B}^{(1)}(\bar{z})\right) .
\end{aligned}
$$

We expect such a form to be also invariant around 1 without any additional constraint on the $\alpha_{i}$. Again, because there is no solution free of logarithms one expects a $\log ^{2}$ dependence if the physical correlators do not vanish. 


\section{Conclusion}

The current superalgebra approach to gaussian disordered systems provides a unifying framework for determining exponents of the models and correlation functions. The logarithmic dependence of correlation functions at critical points is traced back to indecomposable representations which are common in superalgebras. Chiral correlation functions possess a logarithmic dependence. However for values of the level $k$ for which the theories have good locality properties, the physical operators do not have a logarithmic dependence. This conclusion was not apparent from the start; it came out of imposing crossing symmetry. It is not clear at this stage what is the significance of the logarithmic operators in the context of the disordered systems. If they have vanishing dimension, as is the case here, they might be related to the density of states.

The correlation functions with $\log$ and $\log ^{2}$ dependence provide examples of correlators whose existence was predicted in [16]. The negative conformal weights are natural in this treatment of disorder because one is considering non-unitary theories at $c=0$. It should be also possible to obtain similar results by considering $W Z W$ models of cosets of KacMoody superalgebras.

Acknowledgements: It is a pleasure to thank D. Bernard for many suggestions. We also thank M. Bauer, P. Di Francesco for discussions. Z.M. thanks R. Balian and J.M. Luck for discussions, and D.S. thanks P. Sorba and L. Frappat. We are grateful to S. Nonnenmacher for many discussions.

\section{Appendix}

The non-vanishing components of the three invariant tensors are equal to the components of the three vectors:

$$
\begin{aligned}
I_{1} & =(1144)+(1234) 4 \epsilon \gamma+(1324) 4 \epsilon \gamma-(1414)+(2143) 4 \epsilon \gamma \\
& +(2233) 16 \epsilon^{2} \gamma^{2}+(2323) 16 \epsilon^{2} \gamma^{2}-(2413) 4 \epsilon \gamma+(3142) 4 \epsilon \gamma+(3232) 16 \epsilon^{2} \gamma^{2} \\
& +(3322) 16 \epsilon^{2} \gamma^{2}-(3412) 4 \epsilon \gamma-(4141)-(4231) 4 \epsilon \gamma-(4321) 4 \epsilon \gamma+(4411) \\
I_{2} & =(1234) 4 \epsilon \gamma-(1243) 4 \epsilon \gamma+(1324) 4 \epsilon \gamma-(1342) 4 \epsilon \gamma \\
& -(1414)+(1441)-(2134) 4 \epsilon \gamma+(2143) 4 \epsilon \gamma+(2233) 32 \epsilon^{2} \gamma^{2}+(2323) 16 \epsilon^{2} \gamma^{2} \\
& +(2332) 16 \epsilon^{2} \gamma^{2}-(2413) 4 \epsilon \gamma+(2431) 4 \epsilon \gamma-(3124) 4 \epsilon \gamma+(3142) 4 \epsilon \gamma \\
& +(3223) 16 \epsilon^{2} \gamma^{2}+(3232) 16 \epsilon^{2} \gamma^{2}+(3322) 32 \epsilon^{2} \gamma^{2}-(3412) 4 \epsilon \gamma+(3421) 4 \epsilon \gamma \\
& +(4114)-(4141)+(4213) 4 \epsilon \gamma-(4231) 4 \epsilon \gamma+(4312) 4 \epsilon \gamma-(4321) 4 \epsilon \gamma \\
I_{3} & =(1234)-(1243)+(1324)-(1342)+(1423)+(1432)-(2134)+(2143) \\
& +(2233) 8 \epsilon \gamma+(2314)+(2323) 8 \epsilon \gamma+(2332) 8 \epsilon \gamma-(2341)-(2413)+(2431) \\
& -(3124)+(3142)+(3214)+(3223) 8 \epsilon \gamma+(3232) 8 \epsilon \gamma-(3241)+(3322) 8 \epsilon \gamma \\
& -(3412)+(3421)-(4123)-(4132)+(4213)-(4231)+(4312)-(4321)
\end{aligned}
$$


The matrices $P$ and $Q$ are given by:

$$
P=\left(\begin{array}{ccc}
1 & 0 & 0 \\
-2 & -3 & -\frac{1}{2 \epsilon \gamma} \\
4 \epsilon \gamma & 8 \epsilon \gamma & 1
\end{array}\right) \quad, \quad Q=\left(\begin{array}{ccc}
-1 & 0 & -\frac{1}{2 \epsilon \gamma} \\
2 & 1 & \frac{1}{2 \epsilon \gamma} \\
-4 \epsilon \gamma & -4 \epsilon \gamma & -1
\end{array}\right)
$$

The following tensors are needed in order to implement the crossing-symmetries:

$$
J_{A}^{\alpha_{1} \alpha_{2} \alpha_{3} \alpha_{4}}=(-1)^{\varepsilon_{\alpha_{2}} \varepsilon_{\alpha_{3}}} I_{A}^{\alpha_{1} \alpha_{3} \alpha_{2} \alpha_{4}}, K_{A}^{\alpha_{1} \alpha_{2} \alpha_{3} \alpha_{4}}=(-1)^{\varepsilon_{\alpha_{2}}\left(\varepsilon_{\alpha_{3}}+\varepsilon_{\alpha_{4}}\right)+\varepsilon_{\alpha_{3}} \varepsilon_{\alpha_{4}}} I_{A}^{\alpha_{1} \alpha_{4} \alpha_{3} \alpha_{2}} .
$$

The $\varepsilon_{\alpha_{i}}$ refers to the parity of the index $\alpha_{i}$. One has the following decomposition:

$$
\begin{gathered}
J_{1}=-I_{1}, \quad J_{2}=-I_{1}+I_{2}-4 \epsilon \gamma I_{3}, \quad J_{3}=-I_{3} \\
K_{1}=I_{2}-4 \epsilon \gamma I_{3}, \quad K_{2}=I_{1}-4 \epsilon \gamma I_{3}, \quad K_{3}=-I_{3} .
\end{gathered}
$$

We rewrite this compactly as $J=C_{1} I$ and $K=C_{2} I$ where $C_{1,2}$ are two matrices which are used to implement the crossing symmetry constraints.

\section{References}

[1] J.-S. Caux, I.I. Kogan and A.M. Tsvelik, Nucl. Phys. B466 (1996) 444.

[2] C. Mudry, C. Chamon and X.-G. Wen, Nucl. Phys. B466 (1996) 383.

[3] K. Efetov, Adv. Phys. 32 (1983) 53.

[4] D. Bernard, (Perturbed) Conformal Field Theory Applied to 2D Disordered Systems: An Introduction, lectures presented at the '95 Cargèse Summer School on "Low dimensional application of quantum field theory", preprint SPhT-T95/113, IHES/P/95/85, hep-th/9509137.

[5] V. Dotsenko and Vl. Dotsenko, Adv. Phys. 32 (1983) 129.

[6] A. Ludwig, Nucl. Phys. B330 (1990) 639.

[7] R. Shankar, Phys. Rev. Let. 58 (1987) 2466.

[8] G. Mussardo and P. Simonetti, Phys. Lett. B 351 (1995) 515.

[9] A. Ludwig, M. Fisher, R. Shankar and G. Grinstein, Phys. Rev. B50 (1994) 7526.

[10] P. Ginsparg, Applied Conformal Field Theory, Les Houches lectures, Session XLIX, 1988, "Fields, Strings and Critical Phenomena", Elsevier Science Publishers B.V. (1989).

[11] P. Goddard and D. Olive, Int. J. Mod. Phys. A 1 (1986) 303, and references therein.

[12] M. Scheunert, W. Nahm and V. Rittenberg, J. Math. Phys. 18 (1977) 155.

[13] M. Marcu, J. Math. Phys. 21 (1980) 1277, J. Math. Phys. 21 (1980) 1284. 
[14] V.G. Kac, Adv. Math. 26 (1977) 8-96.

[15] L. Rozansky and H. Saleur, Nucl. Phys. B376 (1992), 461-509.

[16] V. Gurarie, Nucl. Phys. B410 (1993), 535-549.

[17] H.G. Kausch, Curiosities at $c=-2$, preprint DAMTP 95-52, hep-th/9510149;

M.R. Gaberdiel, H.G. Kausch, Nucl. Phys. B477 (1996) 293-318;

M.A.I. Flohr, Int. J. Mod. Phys. A11 (1996) 4147-4172.

[18] M.A.I. Flohr, Mod. Phys. Lett. A11 (1996) 55-68.

[19] V.G. Knizhnik and A.B. Zamolodchikov, Nucl. Phys. B247 (1984) 83-103.

[20] E.L. Ince, Ordinary Differential Equations, Dover Publications Inc. (1956).

[21] Vl.S. Dotsenko and V.A. Fateev, Nucl. Phys. B240 (1984) 312-348. 